\title{
Stimulus novelty and emotion perception: the near absence of habituation in the visual cortex
}

\author{
Harald T. Schuppa , Jessica Stockburger ${ }^{\mathrm{a}}$, Maurizio Codispoti ${ }^{\mathrm{d}}$, Markus Junghöfer ${ }^{\mathrm{b}}$, Almut I. Weike \\ and Alfons O. Hamm ${ }^{c}$
}

\begin{abstract}
${ }^{\mathrm{a} D}$ Department of Psychology, University of Konstanz, Konstanz, ${ }^{\mathrm{b}}$ Institute for Biomagnetism and Biosignalanalysis, Münster University Hospital, Münster,
'Department of Psychology, University of Greifswald, Greifswald, Germany and Department of Psychology, University of Bologna, Bologna, Italy
\end{abstract}

Correspondence and requests for reprints to Dr Harald T. Schupp, Institute of Psychology, University of Konstanz, PO Box D36, 78457 Konstanz, Germany Tel: + 49 753l 882504; fax: + 49 753l 882971; e-mail: Harald.Schupp@uni-konstanz.de

Sponsorship: This work was supported by the German Research Foundation (DFG) Grants Schu 1074/7-3 and I074/I0-I.

Received I0 January 2006; accepted I2 January 2006

In rapid serial visual presentation of pictures, an early event-related brain potential component shows enlarged negativity over occipital regions for emotional pictures compared with neutral pictures. The present study examined whether the processing of emotional target pictures varies as a function of stimulus repetition. Accordingly, pictures of erotica, neutral contents, and mutilations were repeatedly presented (90 times) while the electroencephalogram was recorded with a 129 dense sensor array.

Keywords: attention, emotion, event-related brain potential, habituation
As in previous studies, emotional pictures were associated with a larger posterior negativity than neutral pictures. Furthermore, differential emotion processing did not vary as a function of stimulus repetition and was similarly expressed across blocks of picture presentation. These findings suggest the near absence of habituation in differential emotion processing during perceptual processing. NeuroReport 17:365-369 (C) 2006 Lippincott Williams \& Wilkins.

\section{Introduction}

Growing evidence from electroencephalographic and neuroimaging research demonstrates the preferential processing of emotional stimuli. Functional magnetic resonance imaging (fMRI) revealed increased blood oxygen leveldependent signal in the extrastriate, occipito-parietal, and inferior temporal cortex when viewing emotionally arousing pictures compared to neutral pictures [1-3]. Furthermore, a recent study determined that increased activity to emotional stimuli in the lateral inferior occipital and inferior temporal visual cortex is observed when the stimulus materials are shown at rapid presentation rates [4]. These results concur with recent event-related brain potential (ERP) studies detailing the temporal dynamics of differential emotion processing. When pictures are presented at rapid rates, a difference in processing pleasant and unpleasant, compared to neutral pictures, is shown by a larger early posterior negativity (EPN) developing around $150 \mathrm{~ms}$ after stimulus onset and lasting until about $300 \mathrm{~ms}$. Estimating the generator sources of the differential ERP activity suggested that emotional stimuli receive enhanced processing in occipito-temporo-parietal regions, particularly pronounced for right hemispheric regions $[5,6]$. Interestingly, paying explicit selective attention to specific stimulus features (e.g. color, orientation, or shape) and higher-order categories (animal vs. non-animal) is reflected by differen- tial ERP activity with similar topography and latency $[7,8]$. Accordingly, enlarged EPN amplitudes of emotional stimuli have been considered from the perspective of 'natural selective attention' proposing that attention and perception are in part directed by underlying motivational systems of avoidance and approach [9]. Currently, however, it is not determined to what extent the early differential perceptual processing of emotional pictures depends on stimulus novelty.

The repeated presentation of sensory stimuli prompts habituation, that is, decrement across several response systems, considered to reflect a basic learning mechanism [10]. By studying habituation in the processing of emotional cues, recent studies reveal that several output responses (e.g. skin conductance responses, heart rate, and corrugator muscle activity) depend to a great extent on stimulus novelty [11-13], when these cues are not associated with behavioral consequences. Furthermore, fMRI studies also reported habituation in emotional stimulus processing with repeated stimulus presentation in selected limbic cortical and subcortical regions [14-17]. Several studies observed a rather rapid habituation of amygdala activations with repeated presentation of emotional facial expressions. In addition, the cingulate cortex, hippocampus, and dorsolateral prefrontal cortex also showed habituation effects. These findings contrast with observations that habituation was 
less pronounced in the visual cortex when aversive and neutral pictures were repeated a few times [17]. Thus, habituation effects may differ for the various neural structures implicated in stimulus evaluation and response organization. Here, we pursue this hypothesis focusing on the differential emotion processing indicated by the EPN, which appeared as the first reliable ERP component discriminating the processing of emotional from neutral scenes.

In the present study, dense sensor ERPs were measured while participants passively viewed pleasant pictures depicting erotic scenes, unpleasant images showing mutilated bodies, and neutral control images. The picture set was repeated 90 times in order to explore the effects of stimulus repetition on differential emotion processing indexed by the EPN component. In contrast to responses associated with motor preparation, it was assumed that differential emotion processing at the level of perception and evaluation is mandatory and not sensitive to stimulus repetition.

\section{Materials and methods Participants}

Participants were eight introductory psychology students from the University of Greifswald (four women; 20-34 years, mean age 24.0 years). They received course credits toward their research requirements.

\section{Stimulus materials and procedure}

Stimulus materials included 10 images of erotic couples, 20 neutral pictures showing humans in neutral contexts, and 10 pictures depicting scenes of mutilations drawn from the International Affective Picture System [18]. As the EPN amplitude is differentiating between emotionally arousing and neutral contents, the number of stimuli was equated for the arousal rather than the valence dimension. An unpublished follow-up study replicated the results reported here, equating the number of pictures for the valence dimension. Erotic, neutral, and mutilation pictures were presented in a perceptually random order as a continuous stream without perceivable interstimulus interval for $330 \mathrm{~ms}[4,6]$. The picture set was repeated 90 times in different orders, amounting to a total of 3600 picture presentations.

\section{Apparatus and data analysis}

Electrophysiological data were collected from the scalp using a 129-channel system (EGI; Electrical Geodesics, Inc., Eugene, Oregon, USA). Scalp impedance for each sensor was kept below $30 \mathrm{k} \Omega$, as recommended by EGI systems guidelines. The electroencephalogram was collected continuously in the $0.1-100 \mathrm{~Hz}$ frequency range, with a sampling rate of $250 \mathrm{~Hz}$. Continuous electroencephalogram data were low-pass filtered at $35 \mathrm{~Hz}$ before stimulus synchronized epochs were extracted from $48 \mathrm{~ms}$ before until $330 \mathrm{~ms}$ after picture onset. A statistical approach was applied for artifact correction, including the transformation of the ERP data to an average reference [19].

Separate ERPs were calculated as a function of picture valence and picture repetition. To achieve an acceptable signal/noise ratio, ERPs were averaged over five repetitions of each picture set, resulting in 18 blocks of stimulus exposure. A two-step procedure was used to analyze the modulation of the ERP waveform as a function of affect and stimulus exposure. First, repeated measures analyses of variance (ANOVAs), including the factors Affect (erotic, neutral, mutilation pictures) and Stimulus Exposure (18 blocks), were calculated for each time point after picture onset separately for each individual sensor in order to identify the temporal and spatial characteristics of affective ERP modulation. These waveform analyses were conducted using a significance criterion of $P<0.01$. In order to avoid false positives, significant effects were only considered meaningful when the effects were observed for at least eight continuous data points $(32 \mathrm{~ms})$ and two neighboring sensors, revealing significant affective modulation. Second, to provide a more conventional ERP analysis, the EPN amplitude was scored as mean activity over a time interval from 200 to $300 \mathrm{~ms}$ in anterior and posterior sensor clusters on the basis of outcome of the waveform analyses. The temporo-occipital cluster comprised the following EGI sensors of the net: $56,63,64,65,66,69,70,71,74,75$ (left hemisphere) and 83, 84, 85, 89, 90, 91, 95, 96, 100, 108 (right hemisphere). The fronto-central cluster comprised the following sensors of the net: 7, 12, 13, 21, 29, 30, 31, 35, 36, 37, (left hemisphere) and 5, 105, 106, 107, 111, 112, 113, 117, 118, 119 (right hemisphere). Separate repeated measures ANOVAs including the factors Affect (erotica, neutral, mutilation pictures), Stimulus Exposure (18 blocks), and Laterality (left vs. right) were conducted for temporooccipital and fronto-central sensor clusters. For effects involving repeated measures, the Greenhouse Geisser procedure was used to correct for violations of sphericity.

\section{Results}

To determine the interaction of stimulus exposure and affect, a first analysis calculated repeated measures ANOVAs for each sensor and time point separately. Of primary interest, no significant interaction between the factors Affect and Stimulus Exposure was obtained by single sensor waveform analysis. By replicating previous findings, a highly significant main effect of Affect was, however, revealed over temporo-occipital and fronto-central sites beginning around $160 \mathrm{~ms}$ poststimulus and being most pronounced in the $200-300 \mathrm{~ms}$ time window. These results are illustrated in Fig. 1c for the time region of interest (160-312 ms), summarized in $32 \mathrm{~ms}$ bins for illustrative purposes only. In these analyses, a $P$-level of $<0.05,0.01$, and 0.001 corresponds to a main effect of Affect $F(2,14)=3.7,6.5$, and 11.8 , respectively.

To further detail the differential processing of emotion as a function of picture repetition, conventional repeated measures ANOVAs were calculated for the mean ERP amplitudes across the left and right occipito-temporal sensor clusters. Neither the main effect Stimulus Exposure nor an interaction involving Stimulus Exposure approached significance, $F<1$, NS. As expected, selective emotion processing was readily apparent at occipito-temporal sensor clusters, Affect $F(2,14)=25.8, P<0.0001$. Furthermore, affective modulation was more pronounced over the right hemisphere, Affect $\times$ Laterality $F(2,14)=8.7, P<0.05$. Accordingly, separate post-hoc tests were conducted for left and right temporo-occipital sensor clusters. Specifically, erotic images were associated with a more pronounced posterior negativity than neutral images over the left and right temporo-occipital clusters, $F(1,7)>25.2, P<0.01$. Similarly, pictures of mutilations evoked increased EPN amplitudes compared with neutral images over the left and right 
(a)

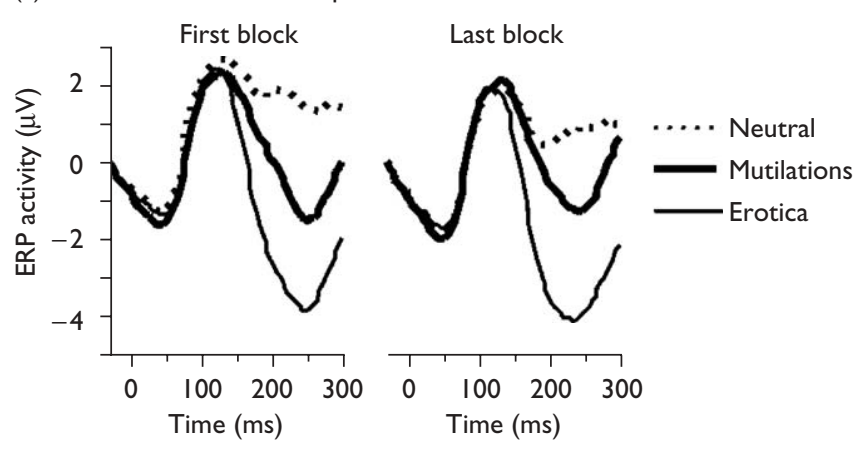

(c)
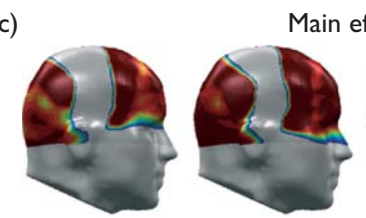

Main effect affect

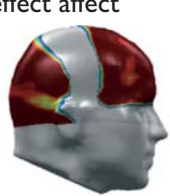

Interaction

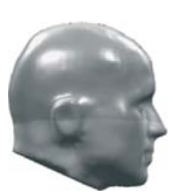

$160-192$

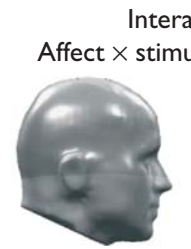

200-232

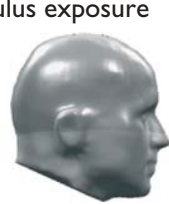

240-272

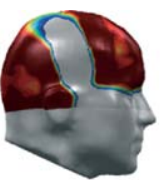

Time (ms)

(b)

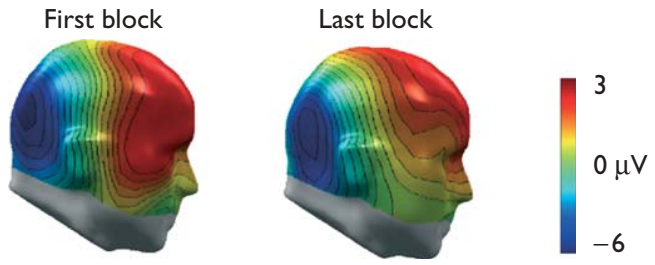

Erotica-neutral
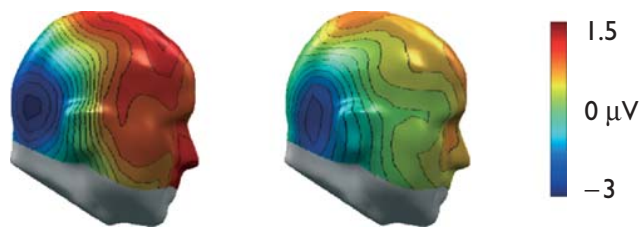

Mutilations-neutral

(d) First block
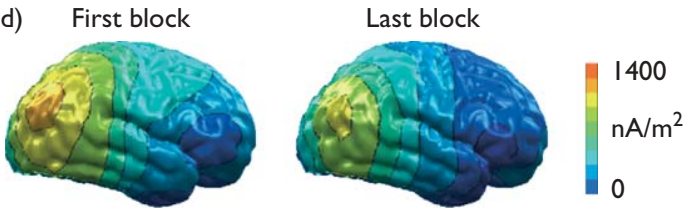

Erotica-neutral
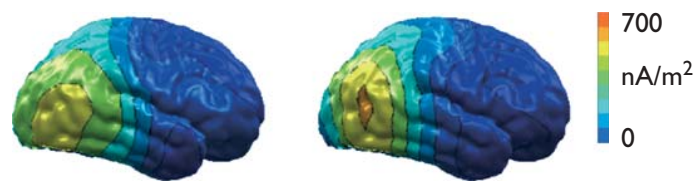

Mutilations-neutral

Fig. I Effects of emotion and stimulus repetition. (a) Grand-averaged event-related brain potential waveforms for a selected right occipital sensor (\#90) as a function of affect and first and last block of picture viewing. (b) Difference scalp potential maps (emotional-neutral) for first and last block of picture presentation separately. (c) Illustration of the statistical effects observed in repeated measure ANOVAs calculated for each sensor and time point shown for the time window of interest (160-312 ms). (d) Calculation of the L2-Minimum-Norm estimate for the scalp potential difference (emotional-neutral) separately for first and last block of picture presentation. All maps display a right-side view.

posterior clusters, $F(1,7)>16.0, P<0.01$. Furthermore, as in previous studies, erotic images elicited more pronounced EPN amplitudes than mutilations over both hemispheres, $F(1,7)>19.8, P<0.01$. As shown in Fig. 2 , a polarity reversal of the EPN effect was observed over fronto-central sites. The statistical effects observed at frontal sites mirrored the findings obtained for the temporo-occipital regions.

Furthermore, as illustrated in Fig. 1a and b, the differential processing of emotional compared with neutral stimuli was similarly pronounced when directly comparing the first and last block of picture viewing, Affect $F(2,14)=34.7, P<0.0001$; again, no interaction of Affect $\times$ Stimulus Exposure was observed $(F(2,14)=1.1, \mathrm{NS})$. Additionally, L2-Minimum-Norm solutions were calculated to provide neural source estimations for the differential processing of emotional cues without the necessity of a priori assumptions regarding the location and/or number of current sources. The calculation of the L2-Minimum-Norm was based on a spherical four-shell isotropic volume conductor head model with 3 (radial, azimuthal, and polar direction) $\times 197$ evenly and spherically distributed dipoles as a source model. A source shell radius of $6 \mathrm{~cm}$ was chosen as a trade-off between depth sensitivity and spatial resolution [20]. As shown in Fig. 1d, differential processing of erotica and mutilation contents was modeled by sources over occipito-temporo-parietal regions, similarly pronounced for the first and last block of picture viewing.

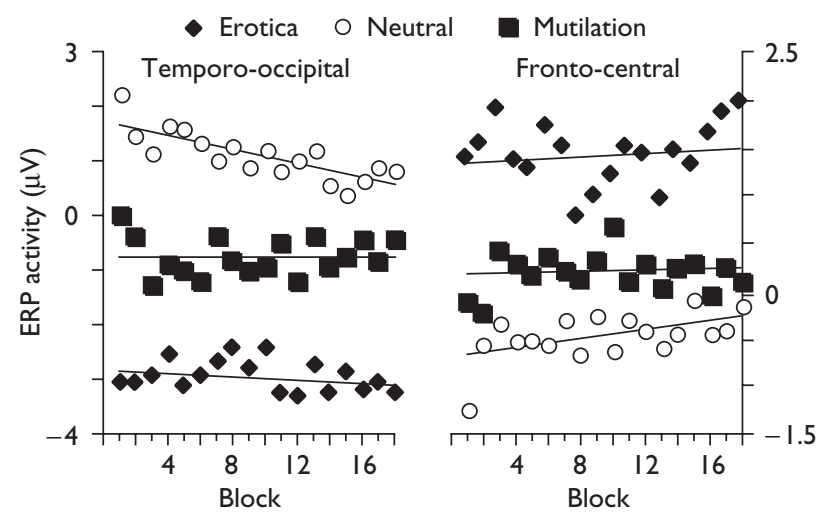

Fig. 2 Mean event-related brain potential activity for right temporooccipital (left panel) and fronto-central (right panel) sensor clusters as a function of affect and stimulus exposure.

\section{Discussion}

The present data replicated previous findings demonstrating enlarged negativity over occipital regions for emotional compared with neutral pictures, particularly pronounced for erotic stimuli $[5,6]$. Building upon this replication, the primary goal of the present study was to explore to what extent the differential processing of emotional cues is dependent on stimulus novelty. Within the context of the present study, results appeared rather clear: repeated 
stimulus presentation did not abolish the differential processing of emotional compared with neutral cues as indexed by the early occipital negativity. These findings suggest that the detection of emotionally significant stimuli in the environment is an obligatory task of perception, evincing little evidence for habituation as a function of passive stimulus experience.

In contrast, response output indices of emotional orienting typically show rapid habituation [11-13]. In these studies, autonomic responses like skin conductance and heart rate as well as facial somatic responses over the corrugator muscle region were selectively engaged when stimuli were novel, but became rapidly habituated after a few stimulus repetitions. A functional analysis of the different facets of affective responses has been suggested to meaningfully explain differences in habituation [13]. Specifically, heart rate is considered to facilitate processes of sensory intake, whereas skin conductance has been associated with preparation for action. With repeated stimulus presentation, neither preparation for action nor continued sensory intake is necessary, and thus, these responses diminish. Each new stimulus, however, needs to be analyzed up to the level of recognition before it can be categorized as previously presented. Thus, facilitated processing of emotional cues during perceptual encoding appeared to be an obligatory process that did not diminish with stimulus repetition.

The perceptual processing in the cortex is suggested to be regulated by subcortical structures involved in appetitive or defensive responding [2,3,21,22]. Recent fMRI studies suggest rather rapid habituation of processing of emotional cues in limbic and paralimbic structures [14-17], which appears inconsistent with the present ERP findings revealing the near absence of habituation of differential emotion processing. A number of critical issues and findings, however, need to be considered. First, it is questionable whether the temporal resolution of fMRI is appropriate to assess the fleeting stage of processing indexed by the EPN. Second, a recent study observed that patients with amygdala lesions did not show enhanced activity to fearful faces in the fusiform and occipital gyrus, which was observed in a group of healthy controls [23]. Third, as observed in a classical conditioning fMRI study, habituation may vary for the various anatomical subregions of the amygdala that perform distinct functions [24]. Fourth, recent studies revealed sizeable blood oxygen level-dependent activations in the amygdala for highly arousing emotional materials used in the present study [2,3], and it remains to be determined in future studies whether the amygdala reveals rapid habituation to these emotionally evocative stimuli. Thus, more research is needed to evaluate the hypothesis that the preferential processing of emotional stimuli in the extended visual cortex is secondary to appraisal of significance in the amygdala. Alternatively, as discussed in associative learning, limbic structures such as the amygdala might modulate the associative strength of cortical stimulus representation of emotionally significant stimuli [25].

\section{Conclusion}

Emotional stimuli receive preferential processing during perceptual encoding presumed to reflect a state of 'natural selective attention' regulated by underlying motivational systems of avoidance and approach. Here, we observed that the early differential processing of emotional compared with neutral stimuli as indexed by the EPN does not depend on stimulus novelty. These findings suggest that detecting emotionally significant stimuli in the environment might be an obligatory task of the organism, apparently not habituating as a function of repeated passive exposure.

\section{Acknowledgements}

We are grateful to Florian Bublatzky for help in collecting these data.

\section{References}

1. Sabatinelli D, Flaisch T, Bradley MM, Fitzsimmons JR, Lang PJ. Affective picture perception: gender differences in visual cortex? Neuroreport 2004; 15:1109-1112.

2. Junghöfer M, Schupp HT, Stark R, Vaitl D. Neuroimaging of emotion empirical effects of proportional global signal scaling in fMRI data analysis. Neuroimage 2005; 25:520-526.

3. Sabatinelli D, Bradley MM, Fitzsimmons JR, Lang PJ. Parallel amygdala and inferotemporal activation reflect emotional intensity and fear relevance. Neuroimage 2005; 24:1265-1270.

4. Junghöfer M, Sabatinelli D, Bradley MM, Schupp HT, Elbert TR, Lang PJ Fleeting images: rapid affect discrimination in visual cortex. Neuroreport 2006; 17:225-229.

5. Junghöfer M, Bradley MM, Elbert TR, Lang PJ. Fleeting images: a new look at early emotion discrimination. Psychophysiology 2001; 38:175-178.

6. Schupp HT, Junghöfer M, Weike AI, Hamm AO. Attention and emotion: an ERP analysis of facilitated emotional stimulus processing. Neuroreport 2003; 14:1107-1110.

7. Potts GF, Tucker DM. Frontal evaluation and posterior representation in target detection. Cognitive Brain Res 2001; 11:147-156.

8. Delorme A, Rousselet GA, Mace MJ, Fabre-Thorpe M. Interaction of topdown and bottom-up processing in the fast visual analysis of natural scenes. Cognitive Brain Res 2004; 19:103-113.

9. Lang PJ, Bradley MM, Cuthbert BN. Motivated attention: affect, activation, and action. In: Lang PJ, Simons RF, Balaban MT, editors. Attention and orienting: sensory and motivational processes. Hillsdale, New Jersey: Erlbaum; 1997. pp. 97-136.

10. Öhman A, Hamm A, Hugdahl K. Cognition and the autonomic nervous system: cognition, anticipation, and cognition. In: Cacioppo JT, Tassinary LG, Berntson GG, editors. Handbook of psychophysiology. 2nd ed. New York: Cambridge University Press; 2000. pp. 522-575.

11. Öhman A, Eriksson A, Fredrikson M, Hugdahl K, Olofsson C. Habituation of the electrodermal orienting reaction to potentially phobic and supposedly neutral stimuli in normal human subjects. Biol Psychol 1974; 2:85-93.

12. Bradley MM, Lang PJ, Cuthbert BN. Emotion, novelty, and the startle reflex: habituation in humans. Behav Neurosci 1993; 107:970-980.

13. Codispoti M, Ferrari V, Bradley MM. Repetitive picture processing: autonomic and cortical correlates. Cogn Brain Res 2006; 1068:213-220.

14. Breiter HC, Etcoff NL, Whalen PJ, Kennedy WA, Rauch SL, Buckner RL, et al. Response and habituation of the human amygdala during visual processing of facial expression. Neuron 1996; 17:875-887.

15. Wright CI, Fischer H, Whalen PJ, McInerney SC, Shin LM, Rauch SL. Differential prefrontal cortex and amygdala habituation to repeatedly presented emotional stimuli. Neuroreport 2001; 12:379-383.

16. Feinstein JS, Goldin PR, Stein MB, Brown GG, Paulus MP. Habituation of attentional networks during emotion processing. Neuroreport 2002; 13:1255-1258.

17. Phan KL, Liberzon I, Welsh RC, Britton JC, Taylor SF. Habituation of rostral anterior cingulate cortex to repeated emotionally salient pictures. Neuropsychopharmacology 2003; 28:1344-1350.

18. Center for the study of Emotion and Attention [CSEA-NIMH]. The international affective picture system: digitizes photographs. Gainesville, Florida: The Center for Research in Psychophysiology, University of Florida; 2001

19. Junghöfer M, Elbert T, Tucker DM, Rockstroh B. Statistical control of artifacts in dense array EEG/MEG studies. Psychophysiology 2000; 37:523-532. 
20. Hank O, Keil A, Elbert T, Müller MM. Comparison of data transformation procedures to enhance topographical accuracy in time series analysis of the human EEG. J Neurosci Methods 2002; 113:111-123.

21. Davis M, Whalen PJ. The amygdala: vigilance and emotion. Mol Psychiatry 2001; 6:13-34.

22. Derryberry D, Tucker DM. The adaptive base of the neural hierarchy: elementary motivational controls on network function. In: Dienstbier RA, editor. Nebraska symposium on motivation, 1990: perspectives on motivation. Lincoln, Nebraska: University of Nebraska Press; 1991. pp. 289-342.
23. Vuilleumier P, Richardson MP, Armony JL, Driver J, Dolan RJ Distant influences of amygdala lesion on visual cortical activation during emotional face processing. Nat Neurosci 2004; 7: 1271-1278.

24. Morris JS, Büchel C, Dolan RJ. Parallel neural responses in amygdala subregions and sensory cortex during implicit fear conditioning. Neuroimage 2001; 13:1044-105.

25. Buchel C, Dolan RJ. Classical fear conditioning in functional neuroimaging. Curr Opin Neurobiol 2000; 10:219-223. 